\title{
High-Frequency Noise in RF Active CMOS Mixers
}

\author{
Payam Heydari \\ Department of EECS \\ University of California, Irvine \\ Irvine 92697-2625
}

\begin{abstract}
A new analytical model for high-frequency noise in RF active CMOS mixers such as single-balanced and double-balanced architectures is presented. The analysis includes the contribution of non-white gate-induced noise at the output as well as the spot noise figure (NF) of the RF CMOS mixer, while accounting for the non-zero correlation between the gate-induced noise and the channel thermal noise. The noise contribution of the RF transconductor as well as the switching pair on the output noise is discussed. The analytical model predicts that the output noise and NF are both a strong function of the $L O$ frequency at gigahertz range of frequencies. Simulation results verify the accuracy of the analytical model.
\end{abstract}

\section{INTRODUCTION}

Mixers in radio-frequency (RF) transceivers are considered as nonlinear blocks. The frequency-varying bias-points of active switching devices due to the large-signal behavior of mixers cause the mixer noise to become essentially a cyclostationary stochastic process. In addition, the wireless standards such as bluetooth and IEEE802.11b operating in multi-gigahertz range demand front-end circuits including the low-noise amplifier (LNA) and the mixer to operate at giga-hertz range of frequencies. The low-frequency analytical models for the mixer noise proposed in [1] and [2] are, therefore, incapable of accurately predicting the noise characteristics of the mixer such as the noise figure (NF), the output noise, or the input-referred noise. More precisely, at RF frequencies, the random potential fluctuations in the conducting channel of a MOS transistor are coupled to the gate terminal through the oxide capacitance and cause a gate noise current even all terminal voltages of the MOS device are fixed. Known as gate-induced noise, this noise source is correlated to the channel thermal noise and its power spectral density (PSD) increases with frequency, hence it is a non-white random process. [3] proposed a stochastic differential equation approach to characterize the noise in CMOS switching mixers. [3] was able to consider the cyclostationary noise generation in mixers. However, this model cannot efficiently analyze the noise in an active mixer in which the switching pair is mostly operating in the triode region whereas the RF transconductor operates in the saturation region.

In this paper we present a high-frequency noise model for the active CMOS mixers while considering the short-channel effects in MOS devices in nanometer technologies. The proposed analytical model is simplified to the noise model presented in [1] at low frequencies. Closed-form expressions resulting from the analytical model gives circuit designers an understanding of how the high-frequency noise affects the mixer NF and output noise.

Section 2 briefly reviews single and double-balanced mixers, and the dominant device noise sources in high-frequencies. Section 3 presents the noise analysis of the CMOS mixer and illustrates the noise contribution of the transconductor and switching pair on the output noise and the NF. Simulation results are provided in Section 4.

\section{BACKGROUND}

\section{A. Large-Signal Analytical Model for RF CMOS Mixers}

Figs 1 (a) and (b) depict the double-balanced and single-balanced cells, respectively, that are extensively used to implement active switching mixers.

To achieve an accurate analytical model for an RF CMOS mixer in nanometer technology, the I-V characteristics of a short-channel device is utilized [4]:

(a)

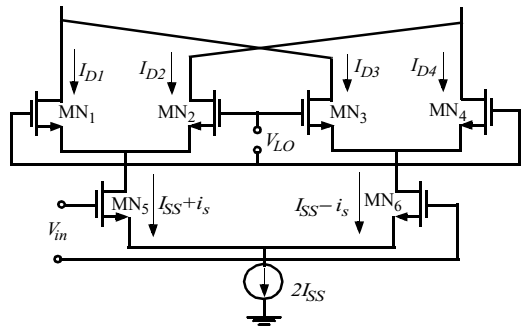

(b)

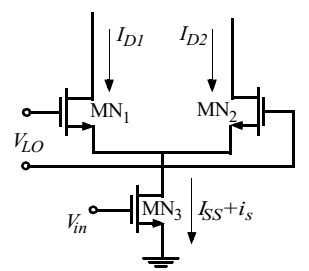

Fig. 1. Active blocks used in switching mixers, (a) a CMOS Gilbert cell. (b) a differential pair.

$$
I_{D}=W v_{s a t} C_{o x} \frac{\left(V_{G S}-V_{T H}\right)^{2}}{V_{G S}-V_{T H}+L E_{s a t}}
$$

where $W$ and $L$ are the transistor channel width and length, respectively, $C_{O x}$ is the gate-oxide capacitance, $v_{s a t}$ is the saturated velocity, and $E_{\text {sat }}$ is the saturated electric field. Using Eq. (1), the ratio of the instantaneous transconductance $g_{m}$ of a short-channel transistor in terms of its DC bias current $I_{D Q}$ is:

$$
g_{m}=\frac{2 I_{D Q}}{V_{G S}-V_{T H}}\left[\frac{\left(V_{G S}-V_{T H}\right) / 2+L E_{s a t}}{V_{G S}-V_{T H}+L E_{s a t}}\right]
$$

Since a large LO signal is applied to the switching transistors, the bias points of MN1 and MN2 are not fixed but vary periodically [1]. The output current of the single-balanced mixer shown in Fig. 1 (b) is a function of the instantaneous LO voltage $V_{L O}$ and the current at the output of the tail current $I_{S S}+i_{S}$. Therefore, we have: 


$$
\begin{gathered}
I_{o}=I_{D 1}-I_{D 2}=F\left(V_{L O}, I_{S S}+i_{S}\right)=F\left(V_{L O}, I_{S S}\right)+\frac{\partial F\left(V_{L O}, I_{S S}\right)}{\partial\left(I_{S S}+i_{S}\right)} i_{S} \\
\text { or } \quad I_{o}=p_{0}(t)+p_{1}(t) i_{S}
\end{gathered}
$$

or

where $p_{0}(t)$ and $p_{l}(t)$ are two periodic waveforms [1]. In a Gilbert-cell double-balanced mixer, $p_{0}(t)$ is eliminated because of the presence of two stages of differential signaling. When the absolute value of the LO voltage is less than a certain voltage, $V_{x}$, the tail current is shared between the two switching devices MN1 and MN2. Otherwise, only one stage turns on and the absolute value of $p_{1}(t)$ thus becomes one, as shown in Fig. 2.

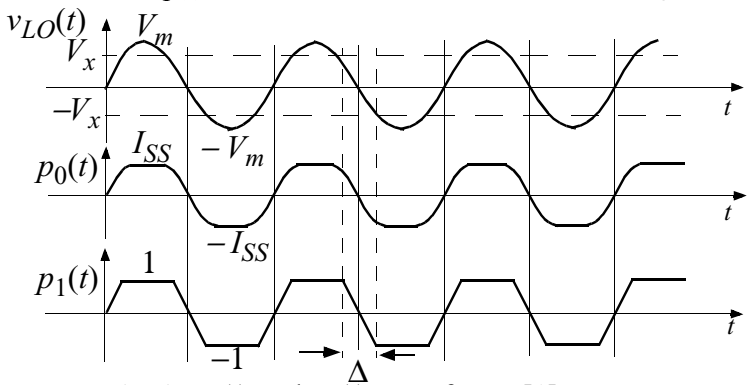

Fig. 2. $p_{0}(t)$ and $p_{1}(t)$ waveforms [1].

\section{B. High-Frequency Noise in MOSFETs}

The ohmic regions of a MOSFET exhibit finite resistivity, thereby contributing thermal noise. In RF integrated circuits, devices with very large channel widths are used. In such cases, the source and drain ohmic resistances are negligible whereas the gate resistance can be significant. The gate resistance forms a distributed RC circuit. However, it can be shown that the distributed effect of the gate resistance in the noise model can be approximated using a lumped resistance whose value is onethird of the physical ohmic gate resistance [5]. The thermal noise due to the ohmic poly resistance is thus as follows:

$$
\overline{V_{n r g}^{2}}=4 K T \frac{r_{G}}{3}
$$

In addition, the thermal fluctuations of channel charge in the MOSFET produce effects that are modeled by drain and gate current noise sources. These current noise sources are partially correlated with each other, because they share a common physical origin and thus possess a spectral power given by:

$$
\begin{aligned}
\overline{i_{n d}^{2}} & =4 K T \gamma g_{m} \\
\overline{i_{n g}^{2}} & =4 K T \delta g_{g} \\
c & =\frac{\overline{i_{n g} i_{n d}^{*}}}{\sqrt{\overline{i_{n g}^{2} i_{n d}^{2}}}}
\end{aligned}
$$

where $g_{g}=\zeta\left(\omega^{2} C_{G S}^{2}\right) / g_{m}$ is the real-part of gate-to-source admittance, and $\gamma, \delta$, and $c$ are bias-dependent factors. For shortchannel MOSFETs, $\gamma$, and $\delta$ are 2.5, and 3, respectively. Because the value of the correlation coefficient, $c$, in the shortchannel regime is not known at present, we will assume that $c$ remains at its long-channel value, which is $j 0.395$ [6].

\section{NOISE ANALYSIS}

The output noise generated in a mixer has a periodically time-varying statistics. This is because an active mixer with a periodic LO is essentially a nonlinear periodic system and its response to an input stationary noise source is a non-stationary process whose statistical properties are invariant to the timeshift by integer multiples of $T_{L O}$, hence it is modeled as a cyclostationary stochastic process. In a cyclostationary process, the mean and autocorrelation function are both periodic. As a consequence, the power spectral density (PSD) is time-varying.

There are three major noise sources that contribute to the noise at the output of the mixer; noise from the transconductor, noise from the switching stage, and noise coming from LO. Note that each of these three major noise sources can be a combination of constituent device noise sources. A comprehensive high-frequency noise analysis of the RF CMOS mixer in the presence of the three noise sources is given in the following. Throughout the forthcoming analysis, we assume that $\Delta f=1 \mathrm{~Hz}$ to simplify the noise expressions.

\section{A. Noise from the RF Transconductor}

Fig. 3 (a) shows a single-balanced cell along with the inputreferred noise sources due to the noise contribution of $\mathrm{MN} 3$ at the input of the transconductor. From a system-level perspective, the noise signal at the transconductor is multiplied by the switching pair's instantaneous current gain $p_{1}(t)$ and generates a current noise $i_{\mathrm{o} 3}(t)$, as shown in Fig. 3 (b).
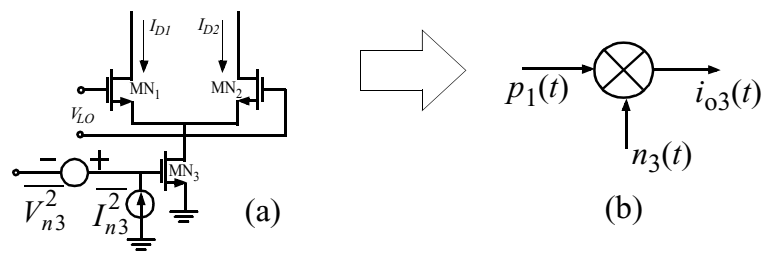

(b)

Fig. 3. (a) Noise contribution of the transconductor in a single balanced mixer, (b) mixer operation for the transconductor's noise.

$$
i_{o 3}(t)=n_{3}(t) p_{1}(t)
$$

$i_{o 3}(t)$ is thus a cyclostationary process due to the fact that $p_{1}(t)$ is a periodic function and $n_{3}(t)$ is a wide-sense stationary process. Subsequently, the output noise PSD of the mixer due to the noise from the transconductor is a time-varying function. However, the time-average of the output noise $i_{o 3}(t)$ is a WSS process [7]. The PSD of this time-average process is [1]:

$$
\overline{S_{n 3}^{o}(\omega, t)}=\sum_{n=-\infty}^{\infty}\left|p_{1, n}\right|^{2} S_{n 3}\left(\omega-n \omega_{L O}\right)
$$

$S_{n 3}(\omega)$ (which is equal to $\overline{i_{n 3}^{2}}$ for $\Delta f=1 \mathrm{~Hz}$ ) is the PSD of the output current noise generated in MN3 whose value is yet to be determined.

To derive $S_{n 3}(\omega)$, the impact of various noise sources must be accounted for. The noise sources contributing on the output noise contain two correlated thermal noise sources, the drain current noise and the gate-induced noise; and uncorrelated noise sources containing the thermal noise of ohmic polysilicon gate, the thermal noise of input source resistance, and the flicker noise. Fig. 4 depicts the high-frequency small-signal model including all these noise sources:

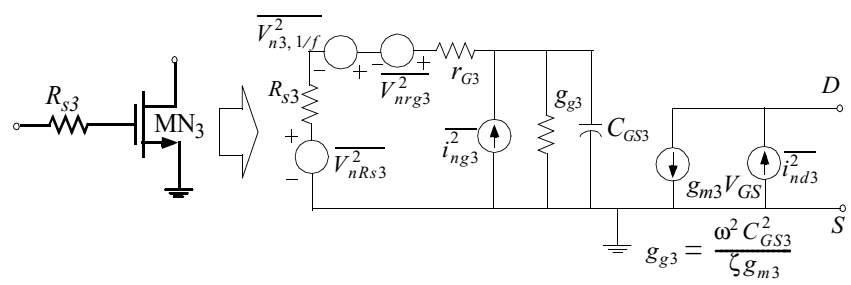

Fig. 4. Noise contribution of the transconductor. 
To quantify the effect of noise at drain node of MN3, the contribution of uncorrelated noise sources are first considered separately. The overall output noise due uncorrelated noise sources is then calculated using superposition. The three uncorrelated noise sources in Fig. 4 are thermal noise of ohmic polysilicon gate $r_{G 3}$, the thermal noise of input source resistance $R_{s 3}$, and the flicker noise. The noise at the output of MN3 due to each uncorrelated noise source is obtained by multiplying its PSD with the square of the transfer function. Table 1 summarizes the derivations for thermal noise of ohmic polysilicon gate and the thermal noise of input source resistance. In the derivation of expressions in Table 1 , it is assumed that $\left(r_{G 3}+R_{s 3}\right)<<g_{m 3} /\left(\zeta \omega^{2} C_{G S 3}^{2}\right)$.

Table 1: Input noise sources and the corresponding PSD of the noise responses at the output of $\mathrm{MN3}$.

\begin{tabular}{|c|c|}
\hline Input noise source & PSD of the noise at the output \\
\hline \hline$\overline{V_{n r g 3}^{2}}$ & $\overline{V_{n r g 3}^{2}} \frac{g_{m 3}^{2}}{\left[\omega C_{G S 3}\left(r_{G 3}+R_{s 3}\right)\right]^{2}+1}$ \\
\hline$\overline{V_{n R s 3}^{2}}$ & $\overline{V_{n R s 3}^{2}} \frac{g_{m 3}^{2}}{\left[\omega C_{G S 3}\left(r_{G 3}+R_{s 3}\right)\right]^{2}+1}$ \\
\hline
\end{tabular}

The overall output noise due to the noise sources, $\overline{V_{n r g 3}^{2}}$ and $\overline{V_{n R s 3}^{2}}$, is frequency-dependent, which, in turn, results in a nonwhite noise at the output of the transistor. To simplify the analytical model, we obtain an equivalent white noise process for this colored-noise process at the output by averaging the noise in the frequency domain over the LO frequency:

$$
S_{n 3}^{u}(\omega) \approx \frac{\left(\overline{V_{n r g 3}^{2}}+\overline{V_{n R s 3}^{2}}\right)}{f_{L O}} \int_{0}^{f_{L O}} \frac{g_{m 3}^{2}}{\left[\omega C_{G S 3}\left(r_{G 3}+R_{s 3}\right)\right]^{2}+1} d f
$$

Calculating the above integral, the overall noise due to the uncorrelated noise sources, therefore, is as follows:

$$
S_{n 3}^{u}(\omega)=4 K T_{m 3}^{2} R_{s G 3}^{\prime}\left(\frac{\omega_{p 3}}{\omega_{L O}}\right) \tan ^{-1}\left(\frac{\omega_{L O}}{\omega_{p 3}}\right)
$$

where $R_{s G 3}^{\prime}=r_{G 3} / 3+R_{s 3}, R_{S G 3}=r_{G 3}+R_{s 3}$, and $\omega_{p 3} \cong\left(C_{G S 3} R_{S G 3}\right)^{-1}$. For the LO frequency much smaller than $\omega_{0},(12)$ is simplified to $S_{n 3}^{u}(\omega)=4 K T g_{m 3}^{2} R_{s G 3}^{\prime}$.

As for the flicker noise, it appears at the output of the mixer around $f_{L O}$ and all the odd-harmonics. The PSD of the flicker noise at the output of the transconductor is found using equations similar to (11) and (12) with the consideration that the flicker noise is a colored noise source.

To account for the partially correlated noise sources, a more complicated analysis needs to be undertaken. Recall that the gate induced noise and drain noise are partially correlated stochastic processes with a correlation coefficient given by (8). This nonzero correlation between the noise sources creates an intricacy in the derivation of the PSD of the output current noise, because the superposition principle is not held valid for the power of correlated stochastic processes. To perform the noise analysis of partially correlated current noise sources, $i_{n g 3}$ and $i_{n d 3}$, the gate induced noise is first expressed as a superposition of its correlated and uncorrelated components [6], i.e.,

$$
\overline{i_{n g 3}^{2}}=\overline{i_{n g 3, u}^{2}}+\overline{i_{n g 3, c}^{2}}=4 K T \delta g_{g 3}\left(1-|c|^{2}\right)+4 K T \delta g_{g 3}|c|^{2}
$$

where $c$ is given by (8). The uncorrelated component, $i_{n g 3, u}$, is treated in the conventional way by obtaining the circuit response using the small-signal transfer function of $\mathrm{MN} 3$. Assuming $\left(r_{G 3}+R_{s 3}\right)<<g_{m 3} / \zeta \omega^{2} C_{G S 3}^{2}$, we have:

$$
\left.S_{n 3}(\omega)\right|_{i_{n g 3, u}}=\left(\frac{g_{m 3}^{2} R_{s G 3}^{2}}{1+\omega^{2} C_{G S 3}^{2} R_{s G 3}^{2}}\right) \overline{i_{n g 3, u}^{2}}
$$

The noise contributions of correlated current noise sources, $i_{n g 3, c}$ and $i_{n d 3}$ on the overall PSD of the drain current of MN3 are determined by obtaining the current response to each individual current noise source, and then computing the mean-square of the resulting summation of these two individual responses. The channel noise source $i_{n d 3}$ directly appears at drain node of MN3. As for $i_{n g 3, c}$, first, the output current of MN3 in response to this current source is obtained. $i_{n g 3, c}$ is fully correlated with $i_{n d 3}$. Therefore, it is treated as a current source proportional to $i_{n d 3}$, i.e.,

$$
\mathrm{F}\left\{i_{n g 3, c}\right\}=\frac{j \omega C_{G S 3}}{g_{m 3}}|c| \sqrt{\frac{\delta \zeta}{\gamma}} \mathrm{F}\left\{i_{n d 3}\right\}=\kappa_{c} \frac{j \omega C_{G S 3}}{g_{m 3}} \mathrm{~F}\left\{i_{n d 3}\right\}
$$

where $\mathrm{F}\{$.$\} represents the Fourier transformation. The total noise$ current at the drain of $\mathrm{MN} 3$ due to $i_{n g 3, c}$ and $i_{n d 3}$ is readily found using small-signal high-frequency circuit for the transistor shown in Fig. 4:

$$
\overline{i_{n c, M N 3}^{2}}=\mathrm{F}\left\{\overline{\left[\left(\kappa_{c}+1\right) i_{n d 3}+\kappa_{c}\left(i_{n d 3} * h_{M N 3}(t)\right)\right]^{2}}\right\}
$$

where $h_{M N 3}(t)=1 / \tau_{G 3} \exp \left(-t / \tau_{G 3}\right.$, and $\tau_{G 3}=C_{G S 3} R_{s G 3}$. Also, $x(t) * y(t)$ stands for the convolution of $x$ and $y$. Using stochastic analysis (the details are omitted due to the lack of space), the overall PSD of the current noise due to the correlated current sources $i_{n g 3, c}$ and $i_{n d 3}$ is as follows:

$$
\left.S_{n 3}(\omega)\right|_{i_{n g 3, c}, i_{n d 3}}=\left[\frac{1+\left(\kappa_{c}+1\right)^{2} \omega^{2} C_{G S 3}^{2} R_{s G 3}^{2}}{1+\omega^{2} C_{G S 3}^{2} R_{s G 3}^{2}}\right] \overline{i_{n d 3}^{2}}
$$

Combining (13) and (17) yields the overall PSD of partially correlated noise sources $i_{n g 3}$ and $i_{n d 3}$ :

$$
\begin{aligned}
\left.S_{n 3}(\omega)\right|_{i_{n g 3}, i_{n d 3}}= & \\
& {\left[\frac{1+\left[\left(\kappa_{c}+1\right)^{2}+\kappa_{c}^{2}\left(\frac{1}{|c|^{2}}-1\right)\right] \omega^{2} C_{G S 3}^{2} R_{s G 3}^{2}}{1+\omega^{2} C_{G S 3}^{2} R_{s G 3}^{2}}\right] 4 K T \gamma g_{m 3} }
\end{aligned}
$$

A plot of $\left.S_{n 3}(\omega)\right|_{i_{n g}, i_{n d 3}}$ vs. frequency is depicted in Fig. 5 .

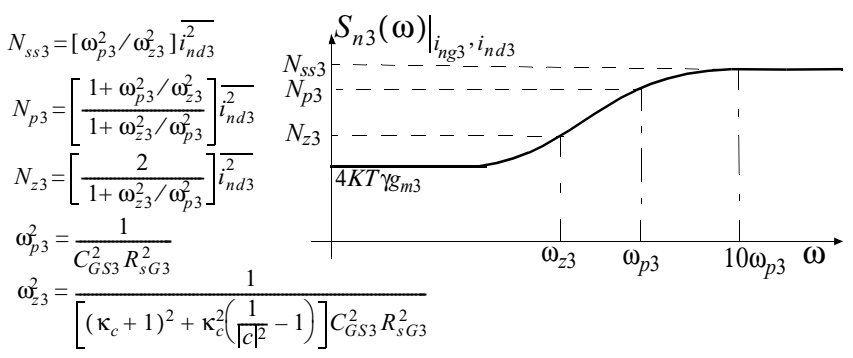

Fig. 5. The PSD of $\left.S_{n 3}(\omega)\right|_{i_{n g}, i_{n d 3}}$ vs. the radian frequency

Direct substitution of $\left.S_{n 3}(\omega)\right|_{i_{n g}, i_{n d 3}}$ in Eq. (10) yields a periodic expansion of $\left.S_{n 3}(\omega)\right|_{i_{n g} 3}, i_{n d 3}$ with a fundamental period $1 / f_{L O}$. However, $\left.S_{n 3}(\omega)\right|_{i_{n g}, i_{n d 3}}$ is frequency-dependent, and hence, a direct substitution does not result in a closed-form expression for the output current noise of the transconductor. To alleviate this problem, we find an equivalent white noise process over the LO frequency for the colored noise component of $\left.S_{n 3}(\omega)\right|_{i_{n g} 3}, i_{n d 3}$. The average power of this equivalent white noise process must be equal to the total area under the colored noise PSD in the interval $\left[0, f_{L O}\right]$ : 


$$
\begin{aligned}
S_{n 3}^{c}(\omega) & =\frac{1}{\omega_{L O}}\left(\left.\int_{0}^{\omega_{L O}} S_{n 3}(\omega)\right|_{i_{n g}, i_{n d 3}} d \omega\right) \\
& =4 K T \gamma g_{m 3}\left[\left(\frac{\omega_{p 3}^{2}}{\omega_{z 3}^{2}}\right)+\left(1-\left(\frac{\omega_{p 3}^{2}}{\omega_{z 3}^{2}}\right)\right)\left(\frac{\omega_{p 3}}{\omega_{L O}}\right) \tan ^{-1}\left(\frac{\omega_{L O}}{\omega_{p 3}}\right)\right]
\end{aligned}
$$

where $\omega_{p 3}$ and $\omega_{z 3}$, defined in Fig. 5, specify the pole and zero of $\left.S_{n 3}(\omega)\right|_{i_{g}, i_{n d}}$, respectively. As expected, the gate-induced noise has a high frequency contribution on the overall noise at the drain node of the transistor. Ignoring the gate-induced noise results in high-frequency error in the noise estimation. In fact, experimental results given in Section 4, verify this observation. The overall PSD of the noise at the output of MN3 is a summation of the output noise due to the correlated noise sources and the output noise due to the uncorrelated noise sources:

$$
\begin{aligned}
S_{n 3}(\omega) & =S_{n 3}^{c}(\omega)+S_{n 3}^{u}(\omega) \\
& =4 K T g_{m 3}\left\{\gamma\left(\frac{\omega_{p 3}^{2}}{\omega_{z 3}^{2}}\right)+\left[g_{m 3} R_{s G 3}^{\prime}+\gamma\left(1-\left(\frac{\omega_{p 3}^{2}}{\omega_{z 3}^{2}}\right)\right)\right]\left(\frac{\omega_{p 3}}{\omega_{L O}}\right) \tan ^{-1}\left(\frac{\omega_{L O}}{\omega_{p 3}}\right)\right\}
\end{aligned}
$$

As a consequence, $S_{n 3}\left(\omega-n \omega_{L O}\right)$ in Eq. (10) becomes frequency-independent. $\overline{S_{n 3}^{o}(\omega, t)}$ is thus simplified to:

$$
\overline{S_{n 3}^{o}(\omega, t)}=S_{n 3}(\omega) \sum_{n=-\infty}^{\infty}\left|p_{1, n}\right|^{2}=E_{p 1} S_{n 3}(\omega)
$$

where $S_{n 3}(\omega)$ is given by Eq. (20), and $E_{p 1}$ is the energy of the Fourier series coefficients of the periodic signal $p_{1}(t)$ and is determined using the Parseval's relation [8]:

$$
E_{p 1}=\sum_{n=-\infty}^{\infty}\left|p_{1, n}\right|^{2}=\frac{1}{T_{L O}} \int_{0}^{T_{L O}}\left[p_{1}(t)\right]^{2} d t
$$

For large LO amplitudes, $p_{1}(t)$ is a periodic square-wave waveform and the average energy per period becomes one.

The overall time-average output noise for the single-balanced mixer shown in Fig. 1 (b) is given by (21). A double-balanced mixer implemented by the Gilbert-cell, should be noisier than a single-balanced mixer because it uses more transistors. A simple extension of the above analysis to the double-balanced mixer results in the following noise expression for the double-balanced mixer of Fig. 1(a):

$$
\overline{S_{n 56}^{o}(\omega, t)}=E_{p 1} S_{n 56}(\omega)=2 E_{p 1} S_{n 3}(\omega)-4 K \operatorname{Tg}_{m 3}^{2} R_{s 3}
$$

\section{B. Noise from the Switching Pair}

The input to the switching pair of an RF mixer is a large LO signal. For a proper circuit operation we assume that $V_{L O}<V_{x}$ (see Fig. 2). Therefore, the current generated by the tail current is shared between the two transistors, and the switching pair stay in the saturation during the entire transition. To capture the highfrequency noise behavior of the differential pair in an RF balanced mixer, the gate induced noise and ohmic gate noise are included in the noise model. When MN1 or MN2 is off, the output current is entirely determined by the current of the tail current and the switching pair does not contribute to the output noise. The noise contributions of MN1 and MN2 become significant during the time interval $\Delta$ indicated in Fig. 2, when the LO signal experiences a signal transition. The overall current noise is a superposition of the individual output noise current associated with each branch side of the differential circuit [1]. The underlying analysis focuses on $I_{D 1}$, noting that a similar analysis can be done for $I_{D 2}$. Similar to the analysis that was undertaken to study the noise behavior of the transconductor, the correlation between the gate-induced noise and the channel noise must be accounted for. Solving the noise equations for the switching pair gives rise to the following equations:

$$
\overline{I_{D 1}^{2}}=\sum_{j=1}^{2}\left(\left.\overline{I_{D 1}^{2}}\right|_{R_{s G j}}+\left.\overline{I_{D 1}^{2}}\right|_{i_{n g j, c}, i_{n d j}}+\left.\overline{I_{D 1}^{2}}\right|_{i_{n g j, u}}\right)
$$

With the aid of the high-frequency circuit model for the transistor, the frequency-average of each term in (24) is:

$$
\begin{aligned}
\left.\overline{I_{D 1}^{2}}\right|_{i_{n g i}, i}= & \frac{4 K T \gamma}{g_{m i}}\left(\frac{g_{m i} g_{m j}}{g_{m i}+g_{m j}}\right)^{2}\left[\left(\frac{\omega_{p 12}^{2}}{\omega_{z 12}^{2}}\right)+\left(1-\left(\frac{\omega_{p 12}^{2}}{\omega_{z 12}^{2}}\right)\right)\left(\frac{\omega_{p 12}}{\omega_{L O}}\right) \tan ^{-1}\left(\frac{\omega_{L O}}{\omega_{p 12}}\right)\right] \\
& \left.\overline{I_{D 1}^{2}}\right|_{R_{s G 12}}=4 K T R_{s G 12}^{\prime}\left(\frac{g_{m i} g_{m j}}{g_{m i}+g_{m j}}\right)^{2}\left(\frac{\omega_{p 12}}{\omega_{L O}}\right) \tan ^{-1}\left(\frac{\omega_{L O}}{\omega_{p 12}}\right)
\end{aligned}
$$

$$
\text { for } i, j=\{1,2\} \text { and } i \neq j
$$

where

$$
\omega_{z 12}^{2}=\frac{\omega_{p 12}=1 /\left(R_{s G 12} C_{G S 12}\right),}{\left[\left(\kappa_{c}+1\right)^{2}+\kappa_{c}^{2}\left(\frac{1}{|c|^{2}}-1\right)\right] C_{G S 12}^{2} R_{s G 12}^{2}}
$$

Similarly, the noise PSD at $I_{D 2}$ is calculated. The output noise is the sum of the PSD at $I_{D 1}$ and $I_{D 2}$, which leads to the following:

$$
\begin{aligned}
& S_{n 12}^{o}(\omega, t)=\overline{I_{D 1}^{2}}+\overline{I_{D 2}^{2}}= \\
& 8 K T\left(\frac{g_{m 1} g_{m 2}}{g_{m 1}+g_{m 2}}\right)\left\{\gamma\left(\frac{\omega_{p 12}^{2}}{\omega_{z 12}^{2}}\right)+\left[\left(\frac{2 g_{m 1} g_{m 2}}{g_{m 1}+g_{m 2}}\right) R_{s G 12}^{\prime}+\gamma\left(1-\left(\frac{\omega_{p 12}^{2}}{\omega_{z 12}^{2}}\right)\right)\right]\left(\frac{\omega_{p 12}}{\omega_{L O}}\right) \tan ^{-1}\left(\frac{\omega_{L O}}{\omega_{p 12}}\right)\right\}
\end{aligned}
$$

Recall that $g_{m 1}$ and $g_{m 2}$ are the instantaneous small-signal transconductances of switching devices MN1 and MN2 in Fig. 1 (b). $g_{m 1}$ and $g_{m 2}$ are periodic signals with a cycle time of $1 / f_{L O}$. The transconductance of the pair, $G(t)=2 g_{m 1} g_{m 2} /\left(g_{m 1}+g_{m 2}\right)$, will thus be a periodic function making $S_{n 12}^{o}(\omega, t)$ to be a periodic function of time. The time-average PSD of $S_{n 12}^{o}(\omega, t)$ gives a time-invariant PSD of the switching pair, i.e.,

$$
\begin{aligned}
& \overline{S_{n 12}^{o}(\omega, t)} \cong \\
& 4 K T\left(\frac{2 I_{S S}}{\pi V_{0}}\right)\left\{\gamma\left(\frac{\omega_{p 12}^{2}}{\omega_{z 12}^{2}}\right)+\left[\left(\frac{1.16 I_{S S}}{\pi V_{x}}\right) R_{s G 12}^{\prime}+\gamma\left(1-\left(\frac{\omega_{p 12}^{2}}{\omega_{z 12}^{2}}\right)\right)\right]\left(\frac{\omega_{p 12}}{\omega_{L O}}\right) \tan ^{-1}\left(\frac{\omega_{L O}}{\omega_{p 12}}\right)\right\}
\end{aligned}
$$

The output noise contribution of the double-balanced mixer is twice the value given by (28).

To obtain the flicker noise contribution from the switching pair, a similar analysis is performed with the consideration that the flicker noise is a colored-noise source.

The noise at the output of the local oscillator has a cyclo-stationary behavior. This is due to the fact that LO is a periodically time-varying circuit. The device noise sources in an oscillator are directly contributing to the phase and amplitude noise of an oscillator [9]. As a simplified treatment, it is assumed that the noise present at the LO port is stationary. The time-average PSD of the current noise at the output of the mixer due to this noise component is:

$$
\overline{S_{n L O}^{o}(\omega)} \cong 4 K T\left(R_{L O}+2 r_{G 1}\right) \overline{G^{2}(t)}=4 K T\left(R_{L O}+2 r_{G 1}\right)\left(\frac{2.32 I_{S S}^{2}}{\pi V_{0} V_{x}}\right)
$$

where $R_{L O}$ is the equivalent noise resistance and $r_{G 1}$ is the physical poly gate resistance.

\section{Noise Figure}

In RF mixers, the image signal does not carry useful information. Therefore, single-sideband noise figure (SSB NF) is preferred to double-sideband noise figure (DSB NF). Employing the SSB NF definition [10], and using equations (22), (28), and (29) in the SSB NF noise definition result in the following NF equations for single and double-balanced mixers: 


$$
\begin{aligned}
N F_{S S B} & =\frac{\int_{0}^{\infty} \overline{S_{n}^{o}(\omega, t)} d \omega}{g_{c}^{2}} \cdot \frac{1}{4 K T R_{s}} \\
& =\frac{\overline{S_{n 3}^{o}(\omega, t)}+\overline{S_{n 12}^{o}(\omega, t)}+\overline{S_{n L O}^{o}(\omega, t)}+4 K T / R_{L}}{g_{c}^{2}} \cdot \frac{1}{4 K T R_{s}} \quad \text { Single-balanced } \\
& =\frac{\overline{S_{n 56}^{o}(\omega, t)}+2 \overline{S_{n 12}^{o}(\omega, t)}+2 \overline{S_{n L O}^{o}(\omega, t)}+4 K T / R_{L}}{g_{c}^{2}} \cdot \frac{1}{4 K T R_{s}} \text { Double-balanced }
\end{aligned}
$$

\section{SIMULATION RESULTS}

A single-balanced mixer was designed and simulated in $0.18 \mu \mathrm{m}$ CMOS process. To include the gate-induced noise parameters in the simulations, the BMIS4 level 54 MOS model was utilized. The output noise, and the NF of the mixer was derived using three approaches; HSPICE simulation, the analytical model developed in [1], and the proposed analytical model in this paper. Fig. 6 (a) depicts the results obtained by these three approaches for three distinct LO voltage amplitudes. For lower frequencies, the noise estimations given by this paper simplifies to those given by [1]. As LO frequency goes beyond few hundred $\mathrm{MHz}$, [1] results in large error because gate-induced noise becomes dominant. Fig. 6 (b) depicts the NF variation with respect to the LO frequency. As clearly seen from Figs. 6 (a) and (b), [1] is incapable of predicting the frequency-dependence of the total output noise and the NF at high frequencies, whereas the proposed noise model is accurately following the simulations. Fig. 7 demonstrates the variation of the noise contribution of the switching pair in terms of the RF transconductor for the designed single-balanced mixer at the LO frequency of $2.4 \mathrm{GHz}$.
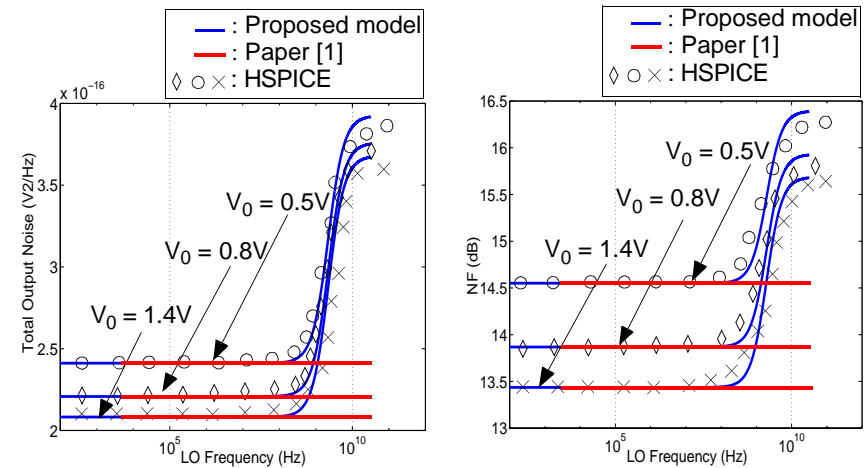

Fig. 6. (a) Total output noise vs. LU trequency, (b) the noise figure $(\mathrm{dB})$ vs. the LO frequency

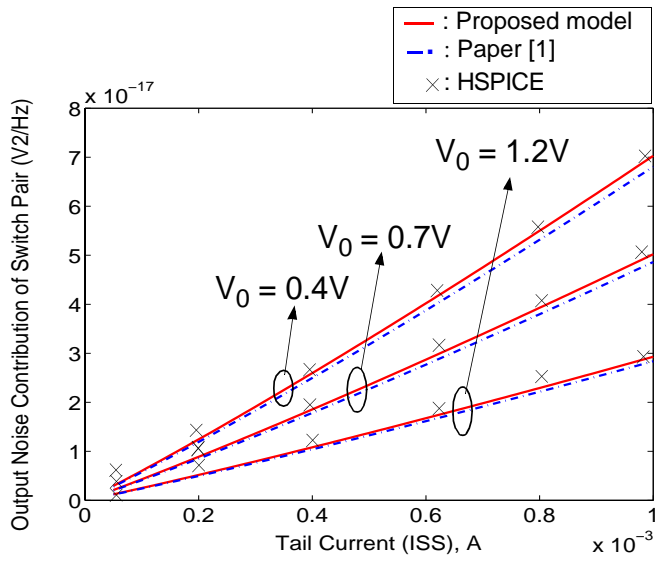

Fig. 7. Switching pair output noise contribution vs. tail current

\section{CONCLUSIONS}

A new analytical model for high-frequency noise in RF active CMOS mixers was presented. The analysis included the contribution of non-white gate-induced noise at the output and the spot noise figure (NF) of the RF CMOS mixer, while accounting for the non-zero correlation between the gate-induced noise and the channel thermal noise. The analytical model predicts that the output noise and NF are both a strong function of the LO frequency at gigahertz range of frequencies. Simulation results verify the accuracy of the analytical model.

\section{REFERENCES}

[1] M. T. Terrovitis, R. G. Meyer, "Noise in Current-Commuting CMOS Mixers," IEEE J. Solid-States Circuits, vol. 34, No. 6, pp. 772-783, June 1999.

[2] H. Darabi, A. A. Abidi, "Noise in RF CMOS Mixers: A Simple Physical Model," IEEE J. Solid-States Circuits, vol. 35, No. 1, pp. 15-25, Jan. 2000.

[3] D. Ham, A. Hajimiri, "Complete Noise Analysis for CMOS Switching Mixers Via Stochastic Differential Equations," IEEE CICC, pp. 439-442, May 2000.

[4] Y. Tsividis, Operation and Modeling of the MOS Transistor, pp. 440-512, McGraw-Hill, 1999.

[5] B. Razavi, R-H. Yan, K. F. Lee, "Impact of distributed gate resistance on the performance of MOS devices, IEEE TCAS I: Fundamental Theory and Applications, Vol. 41, no. 11, pp. 750754, Nov 1994.

[6] T. H. Lee, The Design of CMOS Radio-Frequency Integrated Circuits, Cambridge University Press, 1998.

[7] Papoulis, A., Probability, Random Variables, and Stochastic Processes, McGraw-Hill, 1991.

[8] Oppenheim, A. V., Willsky, A. S. , Young, I. T., Signals and Systems, Prentice-Hall, Inc., 1983.

[9] A. Hajimiri, T. H. Lee, "A General Theory of Phase Noise in Electrical Oscillators," IEEE I. Solid-State Circuits, vol. 33, No. 2, Feb. 1998.

[10] B. Razavi, RF Microelectronics, Prentice-Hall, 1998. 